\title{
Avaliação de resultados da capacitação via estágios pós-doutorais: breves notas sobre a produção científica em periódicos
}

Pedro Marcos Roma de Castro*

Geciane Silveira Porto**

\section{Resumo}

A pesquisa teve como objetivo avaliar a influência do estágio pós-doutoral sobre a produção científica, notadamente das hard sciences de docentes atuantes em programas de pós-graduação da Universidade de São Paulo (USP). 0 estudo busca a mensuração de variações da produção registrada em periódicos científicos em um panorama ex ante e ex post e encontra-se circunscrito nessas quatro grandes áreas do conhecimento: Biológicas, Engenharias, Exatas e da Terra e Saúde. 0 pós-doutorado pode ser visualizado como um local privilegiado para a complementaridade da capacitação docente enquanto forma de atualização e/ou reciclagem profissional, com a lógica embutida e a expectativa de que haja benefícios, também, para o programa de pós-graduação de origem. Como toda atividade de pesquisa não pode ficar implícita, as atividades de pesquisa do pós-doutorado, também, necessitam ser tornadas públicas via conhecimento externalizado que se traduz no conhecimento cristalizado sob a forma de publicações científicas que são compartilhadas pela comunidade acadêmica. Com uma população de 86 doutores que realizaram pósdoutorado até junho de 2005, os dados levantados com o confronto da produção em Ciência e Tecnologia (C\&T), antes e depois do treinamento pós-doutoral apontam para um comportamento de variação da produtividade em mídia qualificada cujo resultado demonstra uma "homogeneização" relativa entre publicações registradas em periódicos Qualis ' $B$ ' e ' $C$ ', nacionais e/ou internacionais, mas sugere haver diferenças no que tange a uma influência sobre o aumento de publicações em revistas Internacionais Qualis 'A' possuindo influência positiva mais visivel sobre essa produção para estágios realizados no Exterior, nas grandes áreas do conhecimento investigadas.

Palavras-chave: Estágio pós-doutoral. Produção científica. Ciência e tecnologia. Pós-graduação e produtividade.

* $\quad$ Doutorando em Administração Geral, Universidade de São Paulo (USP); Analista em Ciência e Tecnologia Pleno da Coordenação de Aperfeiçoamento de Pessoal de Nivel Superior (CAPES). E-mail: pedro.castro@capes.gov.br

*** Doutora em Administração, USP; Professora Livre Docente, Faculdade de Economia, Administração e Contabilidade do campus de Ribeirão Preto da USP (FEA-RP/USP). E-mail: geciane@usp.br 


\section{Evaluation of Outputs of Post-doctoral Research: Brief notes about the scientific production in journals Abstract}

This research aims to evaluate the influence of the post-doctoral research on scientific production in particular of those 'hard science' professors in graduate studies of the University of São Paulo (USP). The study investigates the variations of registered production in scientific Journals in an before and after 'PostGraduate' and it is circumscribed in four great areas of knowledge: Biological, Engineering, Exact and Geosciences and Health. The post-doctoral programs may be seen as an extra privileged space in any complementation of an academic career as a form of bringing up to date and/or professional recycling, with embedded logic and the expectation that the benefits, too, will come to their original postgraduation programs. As all research activity cannot be implied, the research activities of the post-doctorate stage also need to be made known to the public, externalized and translated into crystallized knowledge in the form of scientific publications that are shared by the academic community. This study worked with a population of 86 researchers who held the stage until June/2005 and the results obtained of production in Science \& Technology - S\&T, before and after the postdoctoral training, indicated a relative 'stability' between registered publications in national/international Qualis ' $A$ ' and ' $C$ ' journals of medium and smaller impact, but it also suggested an increase of publications in internationals periodicals Qualis ' $A$ ' with greater impact when sabbaticals or post-doctoral research abroad occur. Keywords: Post-doctoral research. Scientific production. Science and technology. Graduate studies and productivity.

\section{Evaluación de resultados de la capacitación por medio de etapas posdoctorales: breves notas sobre la producción cientifica en revistas Resumen}

Esta investigación tiene por objeto evaluar la influencia de la etapa del posdoctorado sobre la producción científica, específicamente de las hard sciences de profesores de programas de posgrado de la Universidad de San Pablo - USP. La investigación abarca cuatro grandes áreas del conocimiento - Biológicas, Ingenierías, Exactas, Geociencias y Salud - y busca determinar las variaciones de la producción en revistas científicas en el panorama ex ante y ex post. El posdoctorado puede considerarse como un espacio privilegiado para la complementariedad de la formación de profesores en el afán de actualizarse, con la 
lógica integrada de la expectativa de que también existen beneficios para el programa de posgrado de origen. Como toda actividad de investigación no puede ser implícita, así también las actividades del posdoctorado deben venir a público por medio del conocimiento que se traduce en publicaciones científicas compartidas por la comunidad académica. Con una población de 86 doctores que realizaron la etapa posdoctoral hasta junio de 2005, los datos obtenidos de la producción en ciencia y tecnología - C\&T, antes y después de la etapa posdoctoral señalan una estabilidad de las publicaciones en revistas de medio y menor impacto, tanto nacionales como internacionales, $y$, por otro lado, indican que la pasantía trae consigo una tendencia de aumento de artículos en revistas internacionales con mayor impacto y una influencia más visible sobre la producción para la capacitación realizada en el extranjero dentro del ámbito de las áreas del conocimiento analizadas.

Palabras clave: Posdoctorado. Producción científica. Ciencia y tecnología. Posgrado y productividad.

\section{Introdução}

0 discurso contemporâneo recorrente acerca da necessidade de mudança, os rápidos avanços tecnológicos, a instabilidade, a globalização faz parte do cenário internacional e demanda das pessoas, prontidão, qualificação e polivalência. Goergen (1998) aborda que mudanças econômicas e sociais imprimem mudanças na relação existente entre universidade, ciência e sociedade. Hoje a expectativa é que, cada vez mais, se realizem serviços, se produzam conhecimentos e também se formem pessoas capazes de atender aos quesitos de um mundo moldado pela ciência e tecnologia, implicando em consequências para a estrutura administrativa, organizações curriculares e para a própria função e procedimentos acadêmicos.

Nesse panorama, passa a se constituir a otimização da Gestão de Ciência, Tecnologia e Inovação um assunto estratégico das nações, havendo nos últimos anos a necessidade crescente do pais no investimento em Ciência e Tecnologia (CETT). Nesse contexto, também, é compreensão pacífica que a qualificação dos recursos humanos desempenha uma função estratégica no desenvolvimento dos programas de pós-graduação, da ciência, da tecnologia; e, por conseguinte, nas universidades, atualmente, existe um crescente movimento de investimento em qualificação docente tanto em nível de doutorado como de pós-doutorado. Esse movimento tem visado a melhor desempenho do conjunto organizacional e uma melhor resposta institucional à sua missão de ensino, pesquisa e extensão.

A visão é que a capacitação via pós-doutorado age enquanto peça recente na engrenagem da pesquisa, opcional para a carreira acadêmica (CASTRO; PORTO; KANNEBLEY JÚNIOR, 2009; CALVOSA; REPOSSI; CASTRO, 2011), mas que ganha grande destaque nos últimos anos, dentre outros aspectos, dada a sua relevância para o conhecimento, o aprimoramento de competências, a reciclagem profissional e de forma geral a necessidade de atualização. 
Figura 1 - Evolução da procura e concessão de bolsas para realização de Pós-Doc no exterior.

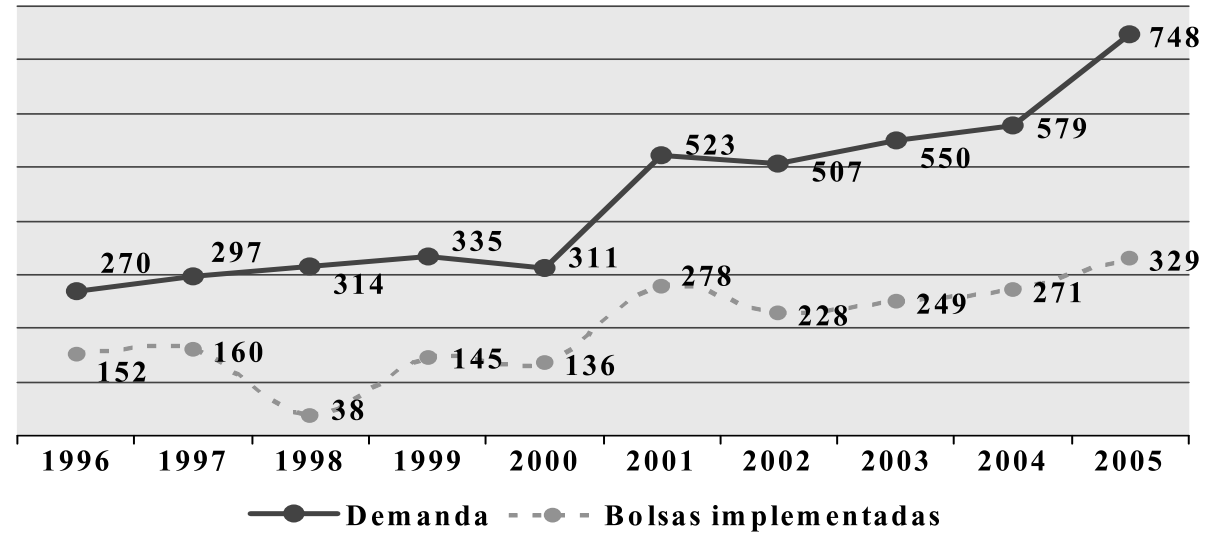

Fonte: Capes (2002).

No que diz respeito a essa nova modalidade inserida na pós-graduação, a Coordenação de Aperfeiçoamento de Pessoal de Nivel Superior (Capes) possui dados registrados até 2005 (Figura 1) que demonstram que, num período de dez anos, se desenha uma demanda crescente em torno dos estágios de pós-doutorado no exterior. Somente no ano de 2000 para 2001, houve um sobressalto da procura, elevando-se em mais de 68\% o interesse e a busca pela realização de cursos/estágios em nível de Pós-Doutoramento, havendo, também, um novo salto da demanda no ano de 2004 para o ano de 2005.

A Figura 1, que aponta para a forte tendência no aumento da demanda, cria a necessidade de recursos públicos para o fomento e, concomitantemente, cria também a necessidade de parâmetros confiáveis e de indicadores de eficiência e eficácia dos estágios pósdoutorais e de sua influência sobre o desempenho dos programas de pós-graduação e/ou a produção científica e tecnológica. 0 balanço é que a conjuntura atual impulsiona a valorização dos programas de capacitação por meio de treinamento em nível de pósdoutoramento, mas também, configura um peso maior à análise do valor final da qualificação funcionando como espaço para a busca de métodos de aferição dos resultados.

Nessa direção de avaliação dos outputs do pós-doutorado, toda a literatura científica expressa em mídia prestigiosa ainda é escassa (CASTRO; PORTO, 2008, 2010; CALVOSA; REPOSSI; CASTRO, 2011) e, por serem bem recentes, mostram ser um campo de exploração em pesquisa em fase 'embrionária'. Esse três estudos são voltados à capacitação para a Ciência, Tecnologia e Inovação, mas com o caráter exploratório focado exclusivamente na produção do conhecimento (Ciência). 0 presente artigo soma nesse cenário, buscando respostas para como age o pós-doutorado e qual a sua possível influência sobre a produção em periódicos, um dos mais importantes estratos dos outputs possiveis na pós-graduação. 
A proposição do pós-doutorado no cenário nacional é uma tentativa de incrementar de forma constante o desenvolvimento científico-tecnológico e da pesquisa, por meio da formação de recursos humanos com qualidade de alto nível, utilizando como instrumento os Pós-Doc, como são "intimamente" denominados os estágios de aperfeiçoamento em nível de pós-doutoramento; e, na Universidade de São Paulo USP, objeto desse estudo, o panorama não se distancia desse quadro atual, representando a maior Instituição de Ensino Superior e o maior sistema de pós-graduação do pais, possuindo um expressivo peso sobre o sistema como um todo.

0 estudo encontra-se circunscrito às grandes áreas de Biológicas, Engenharias, Exatas e da Terra e Saúde e aborda a configuração do "retorno" do estágio pós-doutoral, em termos da produção científica dos docentes atuantes na pós-graduação da USP. A pesquisa trabalhou, basicamente, utilizando como fonte de dados o Sistema Lattes, com a investigação objetiva sobre uma questão emblemática envolta no contexto da realização dos estágios pós-doutorais: 0 Pós-doc contribui para aumentar a produção (publicações) em revistas internacionais indexadas? Haveria diferenças significativas, no desempenho em C\&T, para docentes com pós-doutorados, comparativamente à sua produção científica antes e depois da realização do pós-doutorado?

\section{0 estágio pós-doutoral à luz da Gestão do Conhecimento}

Probst, Raub e Romhardt (2002), colocam que o conhecimento pode ser adquirido de especialistas externos, de outras organizações, de parceiros, de clientes, de vendedores ou de países "produtores" do conhecimento. Vale pontuar que, embora a aquisição do conhecimento seja uma maneira rápida de inserir conhecimento, muitas vezes o conhecimento não está disponível para ser adquirido, o que dá força aos argumentos de criação de Nonaka e Takeuchi (1997) e coloca em pauta a difícil e árdua tarefa relacionada com a necessidade de desenvolver conhecimento nos contextos específicos.

Para Nonaka e Takeuchi (1997), a criação do conhecimento inicia-se no nível do indivíduo, estendendo-se para o grupo e posteriormente para a instituição, podendo atingir niveis interorganizacionais. Essa interação é refletida no Modelo de Conversão, composto por quatro modos: Socialização, Externalização, Combinação e Internalização (Figura 2).

0 conhecimento para Nonaka e Takeuchi (1997) é subdividido em explícito e tácito. A socialização ou a disseminação do tácito ocorre com experiências compartilhadas. Não se trata de um aprendizado por meio da linguagem, mas pela observação via contato social, imitação e prática. Pela externalização, o conhecimento tácito é convertido em explícito, geralmente ocorre com a expressão do conhecimento em metáforas e analogias, que muitas vezes são ineficientes para reproduzir com fidelidade o conhecimento tácito, provocando o diálogo e reflexão coletiva. 
Figura 2 - Esniral do Conhecimento.

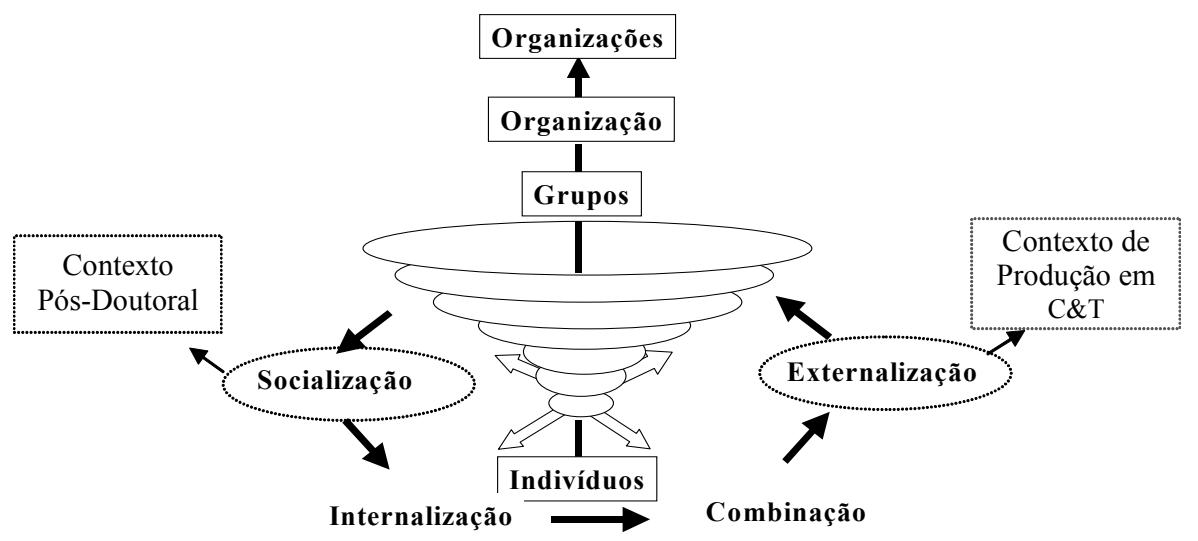

Fonte: Adaptado de Calvosa, Repossi e Castro (2011).

A combinação é a disseminação do explícito baseado na troca de codificáveis e com a internalização, ou conversão do explícito em tácito, o conhecimento é incorporado às bases do indivíduo sob a influência do seu modelo mental e transformado em comportamento. Da interação entre os quatro modos de conversão do conhecimento surge a espiral do conhecimento. A criação do conhecimento se inicia no nivel do indivíduo e, por meio da interação, atinge gradativamente os grupos, equipes, setores, organizações, áreas e redes.

Vista como efeito do coletivo, influenciada pela disponibilidade de informação e qualidade dos canais de comunicação, há uma gama de unidades de conhecimento inter-relacionadas que extrapolam a própria instituição e são geradas por conectividade com outras instituições, universidades ou instituições de pesquisa (ANTONELLI, 1999). Demonstrando que as instituições se beneficiam da proximidade para buscar crescimento por meio de um processo de coevolução, ou seja, evoluir com a produção de conhecimentos inter-relacionados e complementares que extrapolam à própria organização, sendo geradas no caso do pós-doutorado por conectividade com outras universidades ou grupos de pesquisa.

Concebendo-se o pós-doutorado como um processo de interação entre universidades, em que pesquisadores são postos em contatos com instituições relacionadas com o estado da arte de uma determinada área, nota-se que se embute nesse processo uma noção de complementaridade interorganizacional e o pós-doc se situa num espaço em que se mesclam ou se alternam condições de desenvolvimento e de aquisição de conhecimentos via práticas de outros. No processo de interação e de relação entre pesquisadores se estabelecem situações de aprendizagem por meio de conhecimentos peculiarmente tácitos. 
Compartilhar é um dos benefícios amplamente reconhecido pelas relações diretas em uma rede (POWELL; KOPUT; SMITH-DOERR, 1996; AJUHA, 2000). Assim, quando as instituições colaboram para desenvolver uma tecnologia, o conhecimento resultante estará disponivel para todas as organizações parceiras. Então, cada parceiro recebe potencialmente uma quantidade maior de conhecimento, a partir de um projeto conjunto, em relação a um mesmo investimento de pesquisa de forma individual.

0 conhecimento pode ser explorado por meio de reciclagem de conhecimentos existentes que são de domínio individual, resultado de experiências pessoais e em interação com outras pessoas para formação de rede. Esta colocação evidencia o manejo em especial do tácito, enquanto a capacidade de resposta contingencial às demandas específicas de um dado contexto (MCFAYDEN; CANELLA JÚNIOR, 2004). Em função disto, realça-se a importância de se entender relações e interações entre pesquisadores para se compreender estruturas cognitivas expressas nos textos científicos, uma vez que relações tácitas e padrões estruturados de conhecimento constituem fenômenos que são interligados (LEYDESDORFF, 2007).

Isso é bastante salientado por Powell (1998), no momento em que destaca que, em capacitação para o conhecimento, as redes inter-organizacionais propiciam meIhores resultados de inovação em relação às firmas individuais. A complementaridade dos conhecimentos de uma organização, a partir do compartilhamento de informações com outras organizações é evidenciada, também, por Nonaka e Takeuchi (1997), ao apresentar a dimensão ontológica da criação do conhecimento. Diante dessa dimensão, o conhecimento nasce no nível individual, sendo expandido pela dinâmica da interação (socialização do conhecimento) e a dinâmica da espiral do conhecimento.

A 'ampliação' de conhecimentos ou a produção de novos conhecimentos surge quando a interação do conhecimento tácito e explícito se eleva dinamicamente de um nível ontológico inferior até níveis mais altos. Nessa dimensão ontológica, observa-se que o conhecimento só é criado por indivíduos (NONAKA; TAKEUCHI, 1997). Uma rede inter-organizacional, como é o caso que se estabelece no pósdoutorado, não pode criar conhecimentos, mas pode proporcionar um espaço de relações positivas e construtivas entre os atores e o ambiente. Assim, a partilha de informação, de opinião, de colaboração e de mobilização sobre um projeto confrontado às necessidades e ao desconhecido converge para a 'ampliação' do conhecimento das universidades participantes.

Pela externalização, o conhecimento tácito é convertido em explícito. Dessa forma, o estágio pós-doutoral de caráter eminentemente tácito deveria convergir para novas produções científicas e tecnológicas que possuem caráter explícito e podem ser compartilhados e disseminados pela comunidade acadêmica. Mas esse caminho não ocorre de forma tão direta e sem percalços. 
Num artigo polêmico intitulado 'Why Nonaka highlights tacit knowledge: a critical review', Meng Li e Fei Gao (2003) (sem vírgula,) procuram mostrar que o modelo de criação de conhecimento proposto por Nonaka e Takeuchi (1997) é limitado se remetido ao conceito de conhecimento tácito de Polanyi (apud LI; GAO, 2003), proposto originalmente na década de 1950. Os autores da crítica remetem às origens o conceito 'tácito' e apontam que há generalismo no atual conceito de externalização, pois nem todos os conhecimentos tácitos podem vir a ser codificados por meio do simbolismo da linguagem.

0 argumento de Michael Polanyi (1966 apud LI; GAO, 2003) diferencia implicitude de tacitude, ou seja, as capacidades ou potencialidades do conhecimento ser transferido e ensinado. 0 tácito é aquele que não pode ser transferido e que é propriedade de cada indivíduo. Ele está arraigado nas práticas e formas particulares de agir e de conceber as coisas de cada pessoa. Nesse sentido, o tácito pode até vir a ser 'imitado', mas somente o conhecimento com implicitude potencial poderia ser trocado e compartilhado entre os indivíduos via externalização no futuro.

0 pós-doutorado pode ser visualizado como um local privilegiado em que a geração de conhecimentos é vista como resultado de um empreendimento coletivo, fortemente influenciada pela efetiva qualidade dos canais de comunicação entre os agentes aprendizes. Uma das características no processo de partilha de conhecimento entre os pesquisadores, pelo menos no que concerne ao estágio pós-doutoral, é a qualidade eminentemente tácita em que ocorre a interação.

0 'tácito' ou o 'implícito' transfere-se pela socialização, sendo que só é possível quando existe alguma proximidade geográfica. Como o conhecimento está nos individuos que interagem em um ambiente intra- e inter-organizacional, a proximidade possibilita contato, socialização e criação de uma base comum de conhecimento. A transferência de conhecimento depende da proximidade que pode fornecer acesso às redes relacionais locais (COHENDET et al., 1999). Nesse sentido, a lógica do pós-doutorado é incentivar essa 'proximidade geográfica' visando ao processo de desenvolvimento do conhecimento científico e tecnológico por meio da socialização entre pesquisadores em laboratório de primeira linha e relacionados com a produção de conhecimentos de fronteira (CASTRO; PORTO, 2008).

Entretanto, a propriedade da crescente dependência do conhecimento científico das novas oportunidades tecnológicas deve ser vista com ressalva quando se considera a importância do conhecimento tácito para o processo de Ciência, Tecnologia e Inovação. 0 conhecimento científico é codificado e formalizado, podendo ser transferido por outros meios que não a socialização. Neste sentido, ainda que a inovação esteja mais calcada no conhecimento científico, é importante esclarecer que o conhecimento tácito é relevante nos estágios iniciais do desenvolvimento, antes dos padrões terem sido estabelecidos e o design dominante ter sido fixado (DUNNING, 2000). 
Garvin (2001) realiza criticas aos autores centrais da gestão do conhecimento, considerando-os acadêmicos e utópicos no que diz respeito às organizações criadoras do conhecimento. 0 autor sob a idéia de "Três Ms" para a gestão do conhecimento Meaning, Management e Measurement - defende que devam existir ferramentas de mensuração que possibilitem a avaliação da velocidade e dos níveis de aprendizado da organização, uma vez que é impossível gerenciar o que não se pode mensurar.

"0 que não foi dito precisa ser dito em voz alta; caso contrário, não pode ser examinado, aperfeiçoado ou compartilhado" (STEWART, 1998, p. 66). 0 Pós-doutorado com propósitos eminentemente de transferência de conhecimentos científicos e tecnológicos de fronteira por meio da Socialização - nos moldes de Nonaka e Takeuchi (1997), também não pode ou não deveria ficar restrito ao contexto da socialização, mas sim, ao sair do período de 'incubação', não ficar em situações de isolamento, ampliar-se por meio de interação e compartilhamento para processos de Externalização do conhecimento, para que possa vir a ser conhecido pelo demais membros da comunidade científica.

0 pós-doutorado surge no cenário da pós-graduação como um item adicional na carreira acadêmica, que, considerando o atual escalonamento existente, tem seu ponto máximo de exigência o título de doutorado. A idéia inicial é potencializar o cenário da produção e da disseminação científica, com vistas a ampliar a participação dos pesquisadores brasileiros no mainstream da ciência e facilitar a inserção do país na comunidade científica internacional.

0 documento da Capes (2002, p. 1) expõe que:

financia estágio pós-doutoral no exterior de professores e pesquisadores visando ao desenvolvimento de atividades de atualização, cooperação e abertura de novas linhas de pesquisa, sempre inseridas no contexto institucional de atuação do candidato. A natureza das atividades desse programa possui uma perspectiva de cooperação entre professores e pesquisadores, [...].

Velho (2001), na mesma direção, aborda a questão da necessidade de aperfeiçoamento como mola propulsora do Pós-Doutorado. Para a autora, os programas de Pós-Doutorado de fato não vêm sendo de formação, voltam-se mais para reciclagem ou atualização de pesquisadores formados do que para a formação de pesquisadores no sentido estrito do termo. A tendência do Pós-Doutorado é atuar como uma oportunidade de complementação da formação por meio da inserção em um trabalho de pesquisa de ponta, havendo a perspectiva que o trabalho com uma equipe de qualidade estabeleça contato com o estado da arte do mundo científico e abra caminho para publicação em revistas de primeira linha. 
A questão envolta no pós-doc é, então, fomentar o contato de doutores brasileiros com os melhores programas no país ou fora do país, tendo em vista que a formação de recursos humanos de primeira linha requer a aquisição de conhecimentos de fronteira e a socialização em ambientes nos quais ele é gerado, com a expectativa de que com o estágio pós-doutoral, haja benefícios no trabalho e produção docente, bem como benefícios ou impacto positivo para o programa de pósgraduação de origem.

Nesse caminho, o conhecimento explícito é formal e sistemático e, portanto, facilmente comunicado e compartilhado, no caso específico desse estudo, mais facilmente observável e mensurável. 0 conhecimento científico é o conhecimento racional, sistemático, desse modo, tendo-se em perspectiva o pós-doutorado como um processo que visa ao incremento do conhecimento - nesse caso em especial do conhecimento científico - o presente estudo buscou então concentrar os esforços de mensuração em torno do conhecimento externalizado e que se traduz no conhecimento cristalizado sob a forma de publicações científicas em periódicos e que são compartilhadas pela comunidade acadêmica.

\section{Método}

Seguindo padrões nacionais, adotou-se aqui o critério Qualis ${ }^{1}$, utilizado por diversas áreas, que leva em consideração tanto a quantidade (número de publicações) como a qualidade, que expressa pela base de avaliação Capes, em princípio, reflete a qualidade dos veículos ou meios de divulgação científica. No que se refere à indexação, Souza e Paula (2002) abordam que um periódico indexado passa a compor uma base sobre o qual se fundamentarão indicadores bibliométricos, entre eles, o número de citações. Nesse sentido, base e indicadores desenvolvidos pelo Institute for Scientific Information (ISI) e publicados no Science Citation Index (SCl) têm grande aceitação na comunidade científica.

0 fator de impacto do Journal of Citation Report (JCR) também tem boa aceitação nestas quatro áreas investigadas, que o leva em consideração no momento de classificação dos periódicos utilizados para divulgação das pesquisas e trabalhos científicos. Mas, o que se compreende por qualidade é bastante subjetivo sendo que parte dessa avaliação advém da opinião dos 'pares' e do conhecimento que componentes das comissões de área/avaliação têm dos veículos utilizados pelos programas de pós-graduação (SOUZA; PAULA, 2002).

Considerando-se, então, o pós-doutorado como uma comunidade de prática, com ampliação de conhecimentos via socialização; o pós-doc, posteriormente, transporia a situação de socialização (conhecimento tácito) e auxiliaria em incrementos

1 Cabe registrar que a coleta de dados da pesquisa ocorreu anteriormente à (re) estruturação da base Qualis que ocorreu em meados do ano de 2009. 
de publicações científicas (conhecimento explícito). Com esse pressuposto, estruturou-se pesquisa exaustiva e objetiva do que os docentes produziam e realizavam em pesquisa antes do pós-doutorado e o que efetivamente produziram após a realização do estágio em nível do pós-doutorado.

Com a perspectiva do Qualis, o presente estudo trabalhou basicamente utilizando como fonte de dados o sistema Lattes e o efeito investigado foi baseado na variação da produção em periódicos; portanto, vale frisar que a dimensão do efeito investigado neste patamar não cobre efeitos em amplitude sobre o trabalho docente, pois não foram analisados efeitos nos processos de trabalho, ensino, percepção de qualidade do desempenho, motivação pessoal do docente, etc. A dimensão do efeito, neste âmbito, cobre basicamente a produção nas atividades de pesquisa da pós-graduação e produtividade em C\&tT.

A sistemática do Qualis baseia-se na indexação das revistas científicas e na participação qualitativa da comunidade científica, por meio das Comissões de Área da Capes. 0 maior peso ou importância atribuída aos periódicos, mesmo que controverso, justificase pela própria natureza e identidade da pós-graduação stricto sensu. No que tange ao balizamento da base Qualis utilizada no estudo, no que se refere à classificação, especialmente das revistas científicas, adotaram-se dois critérios básicos: a abrangência da circulação (Internacional, Nacional e Local) e niveis de qualidade (A, B, C).

Tabela 1 - Detalhamento da composição das grandes áreas.

\begin{tabular}{l|l|l|l}
\hline Biologia Geral & Eng. Aeroespacial & Astronomia & Educação Física \\
\hline Biofísica & Eng. Biomédica & Computação & Enfermagem \\
Bioquímica & Eng. Civil & Física & Farmácia \\
Botânica & Eng. de Materiais & Química & Fisioterapia \\
Ecologia & Eng. de Minas & Geociências & Terapia Ocupacional \\
Farmacologia & Eng. de Produção & Matemática & Fonoaudiologia \\
Fisiologia & Eng. de Transportes & Oceanografia & Medicina \\
Genética & Eng. Elétrica & Estatística & Nutrição \\
Imunologia & Eng. Mecânica & & Odontologia \\
Microbiologia & Eng. Oceânica & & Saúde Coletiva \\
Morfologia & Eng. Nuclear & & \\
Parasitologia & Eng. Química & & \\
Zoologia & Eng. Sanitária & & \\
\hline
\end{tabular}

Fonte: Capes (2009).

Segundo Souza e Paula (2002) as áreas do conhecimento, incluindo as grandes áreas que compõem a Tabela 1, fazem uso do Qualis e contemplam critérios editoriais, adotando-se os serviços do ISI ou do JCR e, às vezes, 
aplicando-se outros critérios objetivos tais como: tiragem, periodicidade, divulgação, padronização, normalização, etc.. Sobre o que se compreende por qualidade, no interior de cada área, se observa que a classificação de um periódico como $A, B$ ou $C$ ainda envolve componente subjetivo, com a representação dos pares e das comissões de área. Cabe o registro que essa sistemática de classificação sofreu alterações, por meio de um recente documento de caráter normativo Capes (2009) e os veículos periódicos de divulgação científica passaram a ser enquadrados em estratos indicativos da qualidade: $A 1,0$ mais elevado; A2; B1; B2; B3; B4; B5 e C - com peso zero.

A utilização da base Qualis, por envolver a questão qualidade, é essencialmente polêmica, contudo, o presente trabalho utilizou essa base como uma referência e como instrumental para buscar equacionar melhor as publicações científicas em periódicos, adotando-se como procedimento metodológico aplicar a classificação com base nos critérios estabelecidos e adotados à época por cada área, não cabendo na pesquisa aqui os refutar.

\section{População da pesquisa}

A captação de dados relativos à produção acadêmica/docente num panorama ex ante e ex post teve, como fonte principal, a busca em dados secundários, especialmente, tendo por base o sistema Lattes Extrator. A população da pesquisa foi constituída pelos docentes das áreas dispostas na Tabela 1, atuantes na pósgraduação da Universidade de São Paulo que concluíram o pós-doutorado (e/ou que atualizaram essa informação no sistema Lattes) até junho de 2005. 0 levantamento de dados foi realizado de forma censitária, abarcando, sem exceções, todos os docentes que satisfizeram a esse quesito.

\section{Resultados e discussão}

A meta do presente trabalho foi realizar uma avaliação de influência de forma censitária, abarcando todos os docentes pesquisadores do quadro da USP, que atuam nas áreas de Biológicas, Exatas e da Terra, Engenharias e Saúde. Mas, a primeira ressalva nesse quesito é que, apesar de meta censitária, provavelmente alguns docentes não compuseram a presente população por motivos adversos como realização do pós-doc com término após junho/2005, bem como, a possibilidade iminente de defasagem das informações contidas no sistema Lattes, podendo ter havido um gap temporal entre o informado/ alimentado pelo docente e o realizado efetivamente em termos das atividades de qualificação e pesquisa.

Mesmo pressupondo-se a priori que o índice de participação de 100\% não tenha sido efetivamente concretizado, índices bem próximos a esse patamar devem ter 
sido alcançados nessas quatro áreas investigadas. A população de docentes da universidade que realizaram estágios pós-doutorais totalizou, então, 86 docentes pesquisadores, sendo 38 docentes $(44,2 \%)$ do sexo feminino e 48 docentes $(55,8 \%)$ do sexo masculino (Figura 3).

Figura 3 - População pesquisada de acordo com o sexo.

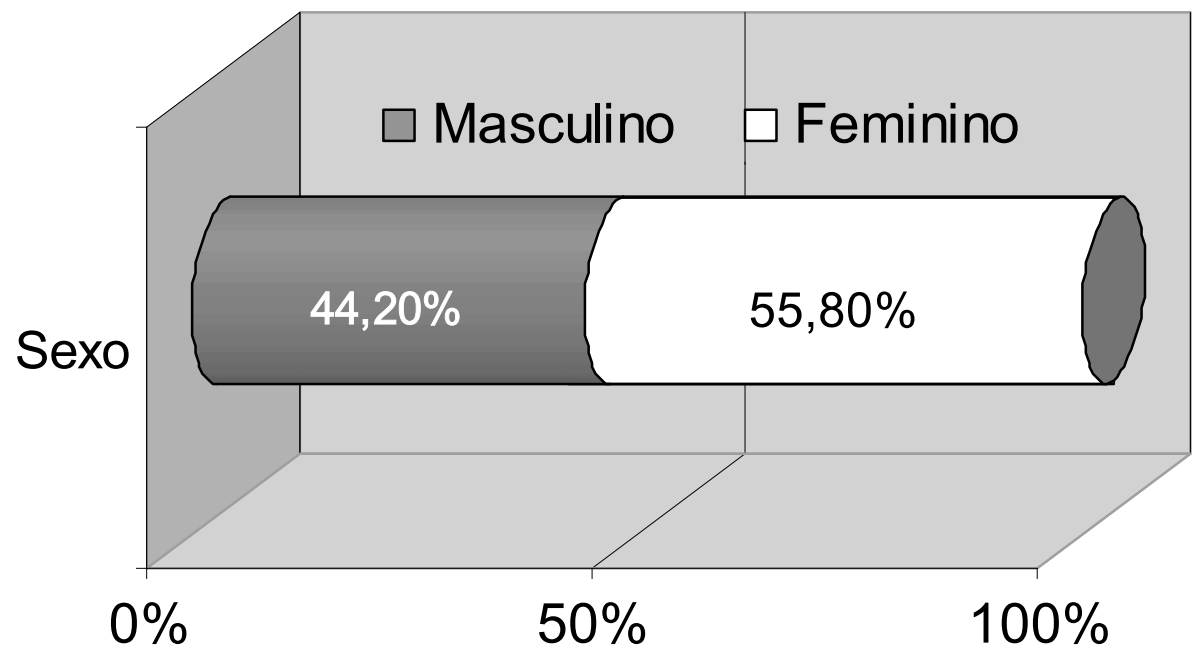

Fonte: As autoras (2010).

A distribuição dos docentes que realizam pós-doutorado e suas respectivas lotações (Tabela 2) demonstrou que quase S! dos pós-docs realizados nessas quatro áreas $(30,2 \%)$ se encontram concentrados nos Institutos de Química e de Física (com 15,1\% cada) do campus da Capital (Química 11,6\%, Física 10,5\%) e do campus de São Carlos (Química 3,5\%, Física 4,7\%), indicando uma maior procura pelos docentes dessas unidades USP para o complemento de estudos por meio do estágio de pós-doutorado. Esses institutos são seguidos, em termos percentuais, pela Escola de Engenharia de São Carlos com 9,3\% e pela Faculdade de Medicina também com 9,3\% (5,8\% na unidade capital - campus extra Cidade Universitária do Hospital das Clínicas na cidade de São Paulo e $3,5 \%$ na unidade do interior - campus de Ribeirão Preto). 
Tabela 2 - Lotação dos docentes com pós-doutorado nas áreas de Biológicas, Exatas e da Terra, Engenharias e Saúde da USP.

\begin{tabular}{l|c|c}
\hline Unidade & Número & Percentual \\
\hline Centro de Biologia Marinha & 1 & $1,2 \%$ \\
\hline Centro de Energia Nuclear na Agricultura & 1 & $1,2 \%$ \\
\hline Escola de Educação Física e Esporte & 3 & $3,5 \%$ \\
\hline Escola de Enfermagem & 1 & $1,2 \%$ \\
\hline Escola de Enfermagem de Ribeirão Preto & 3 & $3,5 \%$ \\
\hline Escola de Engenharia de São Carlos & 8 & $9,3 \%$ \\
\hline Escola Politécnica & 4 & $4,7 \%$ \\
\hline Escola Superior de Agricultura "Luiz de Oueiroz" & 1 & $1,2 \%$ \\
\hline Faculdade de Ciências Farmacêuticas & 2 & $2,3 \%$ \\
\hline Faculdade de Filosofia, Ciências e Letras de Ribeirão Preto & 2 & $2,3 \%$ \\
\hline Faculdade de Medicina & 5 & $5,8 \%$ \\
\hline Faculdade de Medicina de Ribeirão Preto & 3 & $3,5 \%$ \\
\hline Faculdade de Odontologia & 1 & $1,2 \%$ \\
\hline Faculdade de Odontologia de Ribeirão Preto & 1 & $1,2 \%$ \\
\hline Instituto de Astronomia, Geofísica e Ciências & 2 & $2,3 \%$ \\
\hline Instituto de Biociências & 3 & $3,5 \%$ \\
\hline Instituto de Ciências Biomédicas & 5 & $5,8 \%$ \\
\hline Instituto de Ciências Matemáticas e de Computação & 4 & $4,7 \%$ \\
\hline Instituto de Física & 9 & $10,5 \%$ \\
\hline Instituto de Física de São Carlos & 4 & $4,7 \%$ \\
\hline Instituto de Matemática e Estatística & 6 & $7,0 \%$ \\
\hline Instituto de Ouímica & 3 & $11,6 \%$ \\
\hline Instituto de Ouímica de São Carlos & $3,5 \%$ \\
\hline Múcleo de Pesquisa em Neurociências & 1 & $2,3 \%$ \\
\hline Fonteo de Pesquisas Epidemiológicas em Nutrição e Saúde & $1,2 \%$ \\
\hline
\end{tabular}

Fonte: As autoras (2010). 
A distribuição dos docentes com pós-doutorado de acordo com o ano de obtenção do título de doutorado indicou uma distribuição com moda de conclusão do curso de doutorado no ano de 1999, havendo também um segundo pico no número de docentes com título de doutorado obtido no ano de 1997 (Figura 4).

Figura 4 - Distribuição dos docentes com pós-doc e o respectivo ano de obtenção do título de doutorado.

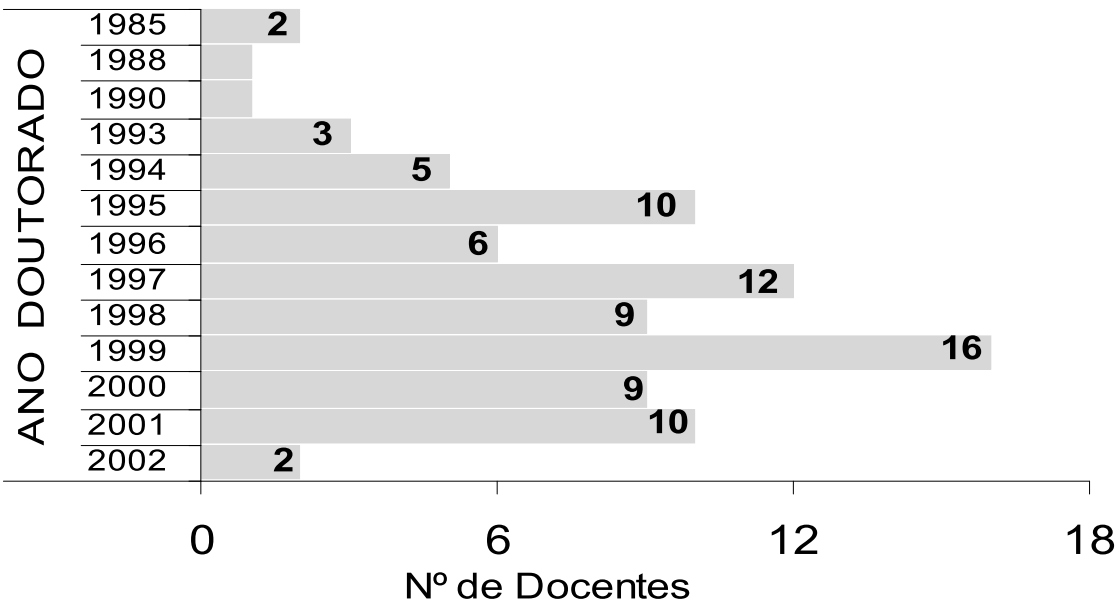

Fonte: As autoras (2010).

A distribuição do ano de conclusão do doutorado dos docentes que realizaram o estágio pós-doutoral nessas quatro áreas na Universidade de São Paulo (Figura 4) possui como média o ano de 1997, com desvio padrão de 3,2 anos, tendo o mínimo de 1985, o máximo de 2002 e uma mediana de 1998. Observa-se uma concentração na porção inferior do gráfico, demonstrando uma distribuição com tendência de concentração da realização do pós-doutorado para os docentes com doutorado concluido em anos mais recentes.

Em relação ao país de realização do pós-doutorado (Figura 5) tem-se a predominância da realização do pós-doutorado no próprio país - 57\% dos casos nessas quatro áreas investigadas - seguido pelo Estados Unidos com 29,1 \% e pela França com $5,8 \%$. No que tange especificamente aos pós-doutorados realizados no exterior, todos os países de realização ou foram da Europa - Alemanha, Espanha, França, Inglaterra e Itália, ou foram da América não-latina - Estados Unidos e Canadá; sendo que, no geral, os Estados Unidos representa sozinho 65,8\% dos estágios pósdoutorais realizados fora do país. 
Figura 5 - Distribuição da escolha dos docentes em relação a0 país de realização do pós-doutorado.

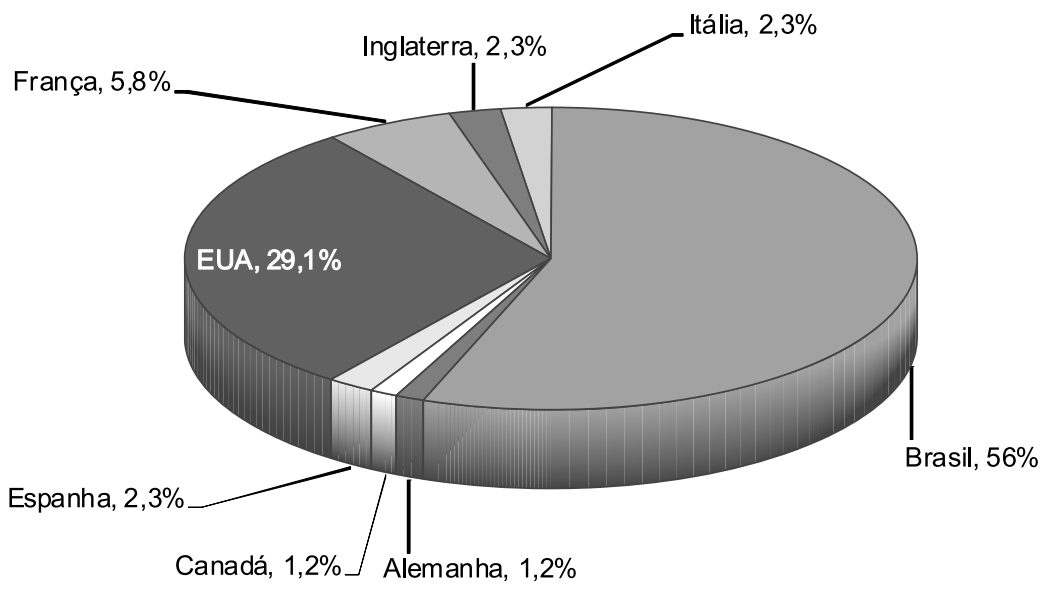

Fonte: As autoras (2010).

Essa relação de predominância dos Estados Unidos fica ainda maior tendo em vista que essa distribuição se encontra diferenciada em função da área do conhecimento. No caso da área de Biológicas e de Engenharias, os Estados Unidos representam 100\% dos casos e os demais paises aparecem apenas nas áreas de Exatas e da Terra e Ciências da Saúde (Tabela 3).

Tabela 3 - Distribuição relativa das áreas em relação aos países dos pós-doc realizados no exterior.

\begin{tabular}{l|c|c|c|c|c}
\hline & Geral & Biológicas & Engenharias & Saúde & Exatas e da Terra \\
\hline Alemanha & $2,6 \%$ & - & - & - & $5,0 \%$ \\
\hline Canadá & $2,6 \%$ & - & - & $8,3 \%$ & - \\
\hline Espanha & $5,3 \%$ & - & - & $16,7 \%$ & - \\
\hline EUA & $65,8 \%$ & $100,0 \%$ & $100,0 \%$ & $50,0 \%$ & $65,0 \%$ \\
\hline França & $13,2 \%$ & - & - & $8,3 \%$ & $20,0 \%$ \\
\hline Inglaterra & $5,3 \%$ & - & - & $16,7 \%$ & - \\
\hline Itália & $5,3 \%$ & - & - & - & $10,0 \%$ \\
\hline Total : & $100,0 \%$ & $100,0 \%$ & $100,0 \%$ & $100,0 \%$ & $100,0 \%$ \\
\hline
\end{tabular}

Fonte: As autoras (2010).

No que diz respeito à produção em periódicos a Figura 6 ilustra a distribuição das publicações, levando-se em consideração as classificações em parâmetros de circulação e qualidade conforme a base Qualis. Nessas áreas, nota-se a predominância das publicações em revistas científicas internacionais consideradas de primeira linha (Internacional A), que representam praticamente a metade das publicações realizadas pelos docentes. 
Figura 6 - Distribuição geral das publicações em periódicos antes e depois do pós-doutorado.

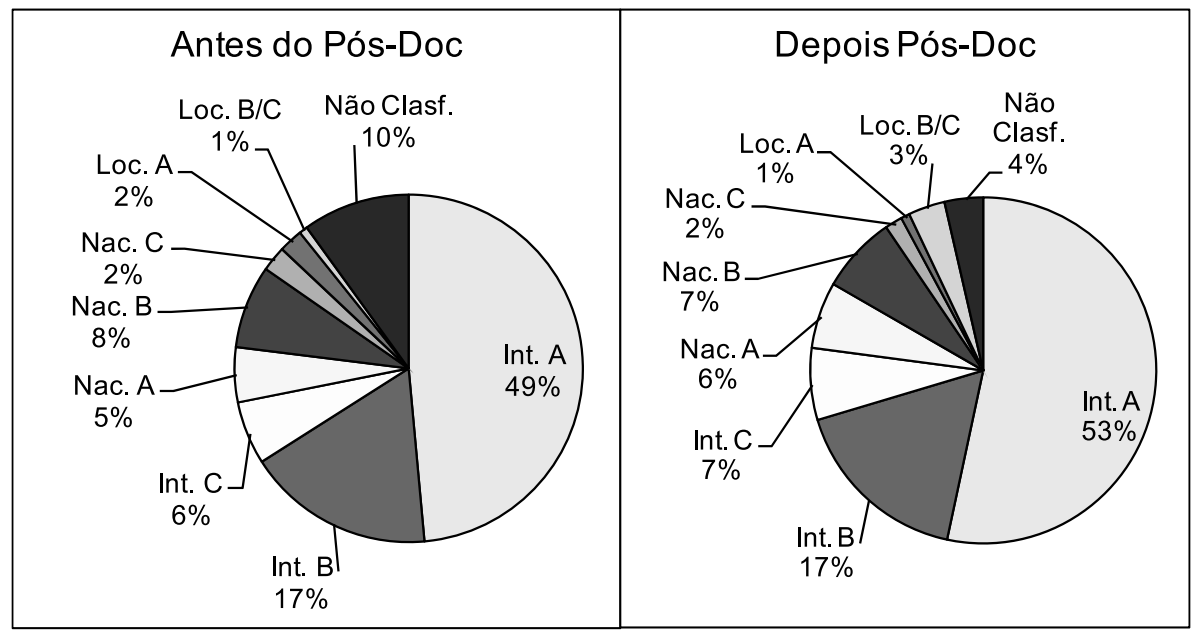

Fonte: As autoras (2010).

Comparando-se os dois gráficos da Figura 6, nota-se que em termos gerais, há um leve acréscimo percentual nas publicações em revistas internacionais A (49\% è 53\%), as demais classificações de revistas internacionais (B e C) e que publicações em revistas nacionais permanecem praticamente constantes e sem alterações expressivas. Verifica-se uma queda acentuada no tocante à produção divulgada em revistas não classificadas ou não avaliadas na base Qualis da Capes (10\% è 4\%), indicando que após o pós-doutorado os docentes passam, então, a encaminhar preferencialmente as produções para as revistas cadastradas e reconhecidas.

Pelo que emerge em relação ao resultado, o pós-doutorado parece contribuir para diminuir o número de publicações em revistas não classificadas e pouco contribui para aumentar a produção (publicações) em revistas internacionais indexadas consideradas 'A'. Mas essa relação mostra-se um pouco mais complexa, os dados da Figura 6 mostram a configuração geral, e essa distribuição sofre a influência de questões como o grande número de doutores da USP que realizaram pós-doutorado no Brasil (conforme Figura 5).

Dividindo-se a população pesquisada em dois grupos os com pós-doutorado no país e os com pós-doutorados realizados no exterior, nota-se que a média de variação das publicações internacionais se mostra de forma diferenciada. Essa divisão entre grupos, no caso específico do treinamento pós-doutoral, faz sentido vez que o acréscimo quantitativo em termos de publicações em periódicos internacionais seria mais pela socialização tácita nos pós-doutorados realizados no exterior e não necessariamente um retorno via socialização realizada com locus no país. 
A variação absoluta da produção para efeitos da Tabela 4 considera e registra a diferença de publicações/ano do docente nos dois momentos (antes e depois), sendo que uma variação média da produção de ordem positiva representa acréscimo em relação ao número de publicações anual que o docente apresentava antes da realização do pós-doutorado. Uma variação de zero significa uma produção estável. Uma variação negativa representa uma queda quantitativa, e assim por diante.

Uma variação negativa na produção implica em dizer que o ritmo de produção acadêmica era maior antes da realização do pós-doutorado, havendo um decréscimo em termos de publicações no cenário ex post. Os resultados indicam (Tabela 4) que se os pós-doutorados visam um start pelo processo de socialização para posterior incremento na produção, os pós-doutorados realizados no Brasil pelos docentes da Universidade de São Paulo - nas áreas de Biológicas, Exatas e da Terra, Engenharias e Saúde - não estão atendendo às expectativas.

A questão do pós-doc realizado no próprio país não estar contribuindo para o aumento do número de publicações em revistas indexadas internacionais coloca a pauta de que, levando-se em consideração o pós-doutorado à luz da gestão do conhecimento, de certa forma esse dado estaria condizente, vez que, a comunidade de prática nesse âmbito ficou circunscrita ao país, e assim, faz sentido que o acréscimo quantitativo em termos de publicações em periódicos internacionais esteja mais voltado para os pós-doutorados no exterior e não para os realizados no Brasil.

Os resultados especificamente para as revistas classificadas no Qualis como Internacionais são dispersos no sentido de que começam a aparecer resultados de influência positiva sobre a produção docente, em alguns casos, mas não em outros, possuindo uma tendência central da variação do índice girar em torno de zero, isto é, produção acadêmica nos mesmos patamares antes e depois da realização do pós-doutorado.

Tabela 4 - Média anual de variação absoluta em número de publicações internacionais após realização do pós-doc.

\begin{tabular}{l|c|c|c|c}
\hline País Pós-Doc & & Internacional C & Internacional B & Internacional A \\
\hline Brasil & Média & 0,0703 & $-0,1261$ & $-0,0809$ \\
\hline & Desvio Padrão & 0,5943 & 0,8212 & 1,9376 \\
\hline Exterior & Média & 0,0293 & 0,1172 & 0,5144 \\
\hline & Desvio Padrão & 0,4116 & 1,0175 & 2,3648 \\
\hline Geral & Média & 0,0522 & $-0,0186$ & 0,2197 \\
\hline & Desvio Padrão & 0,5191 & 0,9156 & 2,2231 \\
\hline
\end{tabular}

Fonte: As autoras (2010).

Nesse sentido, os dados da Tabela 4 demonstram que o pós-doutorado no Brasil não possui influência sobre o quantitativo absoluto das publicações em revistas inter- 
nacionais indexadas e também é inexpressiva para os pós-docs realizados no exterior no tocante as revistas Internacionais ' $B$ ' e Internacionais ' $C$ ', mas essa influência aparece especialmente em relação as revistas Internacionais 'A' que com a média absoluta de $+0,51$ por ano registra que os docentes passam a contar em média a cada dois anos com uma publicação adicional em relação à verificada antes do pós-doutorado.

Os resultados mostram que os pós-doutorados, realizados no exterior, não provocam um incremento generalizado nas publicações internacionais: ele se verifica especialmente para as publicações em revistas internacionais classificadas na base Qualis como "Internacionais A", dado esse que leva a pensar que a influência do estágio sobre a produção em periódicos não se dá de forma geral, mas sim de forma parcial e pontual.

Parcialmente, mas que pode ser interpretado como um resultado 'bem-vindo', pois as revistas Internacionais ' $A$ ' são justamente as revistas que, na nova classificação Qualis, são (re)apresentadas pelo estrato $A 1$, representando os periódicos com elevados fatores de impacto, maior prestígio no meio acadêmico e que possuem grande circulação para além da fronteira de seus paises. Nesse sentido, é desejável que a influência dos pós-doutorados realizados no exterior recaia justamente sobre essa categoria de revistas, pois aumentam as chances de compartilhamento dos conhecimentos externalizados, ampliando as possibilidades de troca e potencializando o caráter público imbuído ao conhecimento advindo da pesquisa científica.

\section{Considerações finais}

No cenário exposto, a presente pesquisa se inseriu na proposição da qualificação docente como parte das discussões sobre os serviços de pesquisa que são executados pela universidade, procurando-se levantar a produção bibliográfica como um aspecto dos estágios em nível de pós-doutorado, por princípio intimamente relacionados com os programas de Pós-Graduação e, por conseguinte, com a Ciência, Tecnologia e Inovação.

Alguns aspectos merecem ser salientados. 0 primeiro é que esse incremento em termos quantitativos das publicações em revistas Internacionais ' $A$ ' não se encontra atrelado a uma redução no que diz respeito a publicações em revistas nacionais de qualidade. As publicações em revistas nacionais se mantêm praticamente constantes, o que significa dizer que o aumento das publicações em revistas internacionais 'A' não comprometeu as publicações em revistas nacionais, fato este que sugere que as revistas Nacionais não foram desprivilegiadas nesse processo e continuaram gozando de prestígio entre os pesquisadores que realizaram pós-doutorado no exterior, tendo em vista que estes continuaram publicando intensamente em revistas 'Nacionais A' e 'B'.

0 outro aspecto é que, em se tratando de Brasil, esse incremento em publicações Internacionais 'A' traz ainda um ponto relevante e positivo no que diz respeito à potencialização do caráter público e de disseminação dos trabalhos de pesquisa. Quase que a totalidade dessas revistas se encontra atualmente (re) classificada como A1 e encontra -se, integralmente, disponibilizada no portal Periódicos Capes (2008), 
com acesso on-line gratuito, o que facilita a disponibilidade desse conhecimento para os professores, estudantes de pós-graduação e a comunidade usuária das bibliotecas nas diversas Instituições de Ensino Superior nacionais.

Chama a atenção, ainda, o fato de mesmo em um estudo com meta censitária a população da pesquisa tenha sido apenas 86 docentes, em se tratando da maior instituição universitária do país. Entretanto, como o corte de pesquisa foi realizado em junho de 2005 (data de atualização do Lattes Extrator), tem-se que, essencialmente, o pós-doutorado é um fenômeno recente que cresce em grande proporções e caso a pesquisa fosse novamente replicada, com o mesmo método e critério para composição da população, o número de pesquisados seria bem maior².

Como importante ressalva, tem-se o fato de que as atividades da pós-graduação não se restringem às atividades de pesquisa. A produtividade aqui mensurada ficou circunscrita a questões vinculadas às atividades de Pesquisa, mas dimensões de Ensino e, também, de Extensão presentes na pós-graduação poderiam ser merecedores de uma atenção especial, sendo esse, um aspecto de limite de escopo do trabalho. Merece destaque que a pesquisa diante da opção de fonte de dados secundários via Lattes, deixou à margem questões como motivação, interesses, duração, reações, percepção de utilidade do estágio, qualidade do ensino, orientações, etc. questões para além dos produtos de pesquisa em periódicos e que poderiam ser ampliadas em pesquisas voltadas para a coleta de dados primários e da percepção dos pesquisadores envolvidos.

Em relação aos resultados em periódicos, há o registro do decréscimo expressivo no que tange às revistas não classificadas na base Qualis, sejam elas locais, nacionais ou internacionais, indicando que os pesquisadores passaram a ver a escolha da mídia de maneira mais criteriosa. No que tange à influência da realização do pós-doutorado sobre as publicações científicas em mídia qualificada, essa não é tão forte e incisiva, mas o resultado que mais se destaca é que o pós-doutorado possui moderada influência sobre o aumento de publicações em revistas Internacionais Qualis A e possui influência mais visível sobre a produção nesse estrato quando realizado no Exterior.

Dessa forma, ao se focar o desempenho e a produtividade em Ciência e Tecnologia, em seu mais importante estrato, as evidências da pesquisa demonstraram que áreas hard sciences da pós-graduação da USP se beneficiaram da proximidade, em especial com instituições no exterior, para buscar por meio do contato e socialização a complementaridade e a ampliação de conhecimentos, neste caso, o conhecimento científico, por meio do processo de co-evolução, ou seja, evoluir a partir de interação com o conhecimento de outras organizações universitárias.

Por fim, na identidade dos interlocutores da gestão do conhecimento, é indispensável reconhecer que há intenção de dar a tônica de que o conhecimento é algo central na

\footnotetext{
2 Em contato dos autores com a Capes, informa-se que em 2008 o número de candidatos interessados foi de 1034 doutores pleiteantes, praticamente o dobro do registrado em 2002 (Figura 1). Em relação a USP, houve aumento expressivo da realização dos estágios somente a partir do final da década de noventa (CASTRO; PORTO, 2010); por assentimento e projeção também espera-se que esse número de realização de estágios esteja configurando-se em patamares quantitativos bem superiores, com comportamento semelhante ao do cenário nacional.
} 
configuração da sociedade contemporânea. No caso do pós-doutorado a ênfase restrita a recursos financeiros, econômicos e/ou tecnológicos não é suficiente para proporcionar a dinâmica e a capacidade da ação universitária no subsistema pós-graduação. Uma das chaves para o aumento de produtividade é o investimento no fator humano, que no caso da pós-graduação se traduz em uma palavra-crucial: os docentes, no sentido de que a produção científica resulta em produtos (livros, artigos...), mas o tempo-espaço do trabalho científico não há separação do produto do ato humano de produção.

\section{Referências}

AJUHA, G. Collaboration networks, structural holes, and innovation: a longitudinal study. Administrative Science Quarterly, New York, v. 45, n. 3, p. 425-455, 2000.

ANTONELLI, C. The evolution of the industrial organization of the production of knowledge. Cambridge Journal of Economics, Oxford. v. 23, p. 243-260. 1999.

CAPES. Orientações para candidatos a estágio pós-doutoral no exterior. Brasília, DF, 2002. Documento não publicado. Disponível em: <http:// www.capes.gov.br/Bolsas/Exterior/Doutoral.htm>. Acesso em: mar. 2004.

Periódicos. Brasilia, DF, 2008. Disponivel em: <http:// www.capes.gov.br>. Acesso em: maio 2009.

. Reestruturação do Qualis: documento do Conselho Técnico Científico. Brasília, DF, 2009. Documento não publicado. Disponível em: $<$ http:// www.capes.gov.br>. Acesso em: maio 2009.

CALVOSA, M. V. D.; REPOSSI, M. G.; CASTRO, P. M. R. Avaliação de resultados da capacitação docente: o pós-doutorado na Universidade Federal Fluminense sob a ótica da produção científica e bibliográfica. Avaliação: revista da avaliação da educação superior, Campinas, SP, v. 16, n. 1, p. 99-122, 2011.

CASTRO, P. M. R.; PORTO, G. S. Análise exploratória sobre avaliação e mensuração de resultados da capacitação via estágios pós-doutorais: heterogeneidade entre grandes áreas do conhecimento?. Revista de Administração, São Paulo, v. 45, n. 1, p. 43-56, 2010.

Retorno ao exterior vale a pena?: a questão dos estágios pós-doutorais sob a perspectiva da produção em C\&t. Organizações \& Sociedade, Salvador, v. 15, n. 47, p. 155-173, 2008.

; KANNEBLEY JÚNIOR, S. Pós-doutorado: essencial ou opcional?. In: ENCONTRO DA ASSOCIAÇÃO NACIONAL DE PÓS-GRADUAÇÃO E PESOUISA EM ADMINISTRAÇÃO, 33., 2009, São Paulo. Anais... São Paulo: ANPAD, 2009.

COHENDET, P. et al. Knowledge coordination, competence creation and integrated networks in globalised firms. Cambridge Journal of Economics, Oxford, v. 23, p. 225-241. 1999. 
DUNNING, J. (Ed.) Regions, globalization and the knowledge economy: the issues stated in regions, globalization and the knowledge-based economy. Oxford: University Press, 2000.

GARVIN, D. Construção da organização que aprende. In: GESTÃo do conhecimento. Rio de Janeiro: Campus, 2001. p. 50-81. (Harvard Business Review Book).

GOERGEN, P. Ciência, sociedade e universidade. Educação \& Sociedade, Campinas, SP, v. 19, n. 63, p. 53-79, 1998.

LEYDESDORFF, L. Scientific communication and cognitive codification: social systems and sociology of scientific knowledge. European Journal of Social Theory, Thousand Oaks, CA, v. 10, n. 3, p. 1-22, 2007.

LI, M.; GAO, F. Why Nonaka highlights tacit knowledge: a critical review. Journal of Knowledge Management, Bradford, UK, v. 7, n. 4, p. 6-14, 2003.

MCFAYDEN, M. A.; CANNELLA JÚNIOR, A. A. Social capital and knowledge creation. Academy of Management Journal, New York, v. 47, n. 5, p. 735-746, 2004.

NONAKA, I.; TAKEUCHI, H. Criação de conhecimentos na empresa: como as empresas japonesas geram a dinâmica da inovação. Rio de Janeiro: Campus, 1997.

POWELL, W. W.; KOPUT, K. W.; SMITH-DOERR, L. Inter organizational collaboration and the locus of innovation: networks of learning in biotechnology. Administrative Science Quarterly, New York, v. 41, n. 1, p. 116-145, 1996.

POWELL, W. W. Learning from collaboration: knowledge and networks in the biotechnology and pharmaceutical industries. California Management Review, Berkeley, v. 40, p. 228-240, 1998.

PROBST, G.; RAUB, S.; ROMHARDT, K. Gestão do conhecimento: os elementos construtivos do sucesso. Porto Alegre: Bookman, 2002.

SOUZA, E. P.; PAULA, M. C. S. Qualis. INFOCAPES: boletim informativo da CAPES, Brasilia, DF, v. 10, n. 2, p. 6-24. 2002.

STEWART, T. A. Capital intelectual. Rio Janeiro: Campus, 1998.

VELHO, L. Formação de doutores no paíse no exterior: estratégias alternativas ou complementares? Dados: revista de Ciências Sociais, Rio de Janeiro, v. 44, n. 3, p. 607-631, 2001.

Recebido em: 16/08/2011

Aceito para publicação em: 28/11/2011 